\title{
Role of cardiac imaging in Anderson-Fabry cardiomyopathy
}

\author{
Walter Serra ${ }^{1 *}$ (D) and Nicola Marziliano ${ }^{2,3}$
}

\begin{abstract}
The Anderson-Fabry disease (AFD, or simply Fabry Disease, FD; MIM \#301500) is a rare X-linked lysosomal storage disorder (Xq22.1) characterized by progressive renal failure, leading to morbidity through cardio- and cerebrovascular involvement. Despite the classic phenotype, only cardiac involvement (cardiac variant of AFD; MIM 301500) is frequent in about $40 \%$ of male and $28 \%$ of female AFD patients, as reported by the Fabry Registry (https://www. registrynxt.com). Morphologically, the cardiac characteristic of the disease, occurs as left ventricular hypertrophy, is accompanied by myocardial fibrosis. Cardiologists may come across these patients during clinical and instrumental evaluation in individuals with non-specific symptoms such as chest pain and arrhythmias, or after instrumental evidence of left ventricular hypertrophy/hypertrophic cardiomyopathy (HCM; MIM 192600). A comprehensive cardiological work-up, including a cardiological visit, a baseline electrocardiogram (ECG) and imaging by both echocardiography $(\mathrm{ECHO})$ and magnetic resonance (MRI) enables identification of the cardiac involvement in patients with a proven diagnosis of AFD. The heart involvement is present in up to 75\% of AFD patients irrespective of their sex. Involvement includes ECG and echocardiography features which suggest AFD and not HCM. Cardiac imaging plays an important role in detecting this sub-type of cardiomyopathy, which, since 2001, has benefited from the introduction of the enzyme replacement therapy (ERT) in symptomatic and pre-symptomatic patients.
\end{abstract}

\section{Introduction}

Many storage diseases are associated with left ventricular cardiac hypertrophy (LVCH; Table 1). Anderson-Fabry disease (AFD) is the most common form of X-linked lysosomal disorder (Xq22.1), with cardiac involvement having a prevalence of $0.5-1 \%$ in patients with hypertrophic cardiomyopathy (HCM; $[1,2])$ typically at the beginning of adulthood. In the classic AFD form (MIM \# 301500), over $60 \%$ of patients of both sexes show cardiovascular signs such as arrhythmias, valve abnormalities and cardiomyopathy. Affected males develop cardiac features around the second decade of life: $\mathrm{LVCH}$, valve diseases and conduction alterations are the earliest signs. Cardiac involvement tends to increase with age, both in incidence and severity, and represents, along with untreated renal failure, the first cause of death among patients with AFD [3, 4].

\footnotetext{
* Correspondence: wserra@libero.it

${ }^{1}$ Cardiology Division, Surgery Department, University Hospital-Parma, Via

Antonio Gramsci 14, 43100 Parma, IT, Italy

Full list of author information is available at the end of the article
}

When should the cardiologist suspect the presence of AFD? Cardiologists may come across such patients in outpatient visits, during clinical and instrumental evaluation in individuals with non-specific symptoms such as chest pain and arrhythmias, or after instrumental evidence of $\mathrm{LVCH} / \mathrm{HCM}$. Generally, once the presence of idiopathic LVCH is confirmed by echocardiography, cardiologists may be orientated towards a classic sarcomeric HCM. However, when investigating family and personal history, asking about extra-cardiac signs and symptoms, and after careful analysis of electrocardiographic traces, cardiologists may be lead to think about non-sarcomeric HCM. Differential diagnosis may be complicated further by the presence of the "cardiac type" of AFD in which only the cardiac phenotype is present, without any other phenotypic traits (corneal opacity, angiocheratoma, hypohydrosis, albuminuria, acroparesthesia), and in presence of a normal renal function [4]. As the disease develops lysosomes are engulfed into metabolite globotriaosylceramide (Gb3) as a result of the absence or partial activity of the a-Gal enzyme. This results in heart conduction disorders (shortening P-wave duration, atrial 
Table 1 Some of the most frequent storage pathologies associated with left ventricular hypertrophy and their chromosomal localization

\begin{tabular}{|c|c|c|}
\hline Disease & MIM & Location (I) \\
\hline CARDIOMYOPATHY, FAMILIAL HYPERTROPHIC, 1; CMH1 & $\# 192600$ & 3p25.3, 1pter-p36.13, 20q11.21 \\
\hline GLYCOGEN STORAGE DISEASE IV; GSD4 & \#232500 & $3 p 12.2$ \\
\hline CARDIOMYOPATHY, DILATED, 1S; CMD1S & \#613426 & $14 q 11.2$ \\
\hline AMYLOIDOSIS, HEREDITARY, TRANSTHYRETIN-RELATED & $\# 105210$ & $18 q 12.1$ \\
\hline HURLER-SCHEIE SYNDROME & \#607015 & $4 p 16.3$ \\
\hline COSTELLO SYNDROME; CSTLO & \#218040 & $11 \mathrm{p} 15.5$ \\
\hline DANON DISEASE & \#300257 & Xq24 \\
\hline MYOPATHY, X-LINKED, WITH EXCESSIVE AUTOPHAGY; MEAX & $\% 310,440$ & $\mathrm{Xq} 28$ \\
\hline FABRY DISEASE & \#301500 & Xq22.1 \\
\hline
\end{tabular}

fibrillation and ventricular tachycardia), cardiac hypertrophy (secondary to lysosomes enlargement) causing LV mild-to-moderate concentric hypertrophy. Moreover, endothelial dysfunction leads to increased risk of myocardial infarction. Cardiac imaging highlights the mechanisms of secondary hypertrophy [5-7]. In addition to lysosomal enlargements, cardiomyocites increase their contractile elements. This hypothesis is supported by the fact that the Gb3 amount does not exceed 3\% of the total myocardial mass, as shown by pathological evidence. The extension of hypertrophy of cardiomyocytes and the accumulation of Gb3, within the vacuums, correlates with the extension of the wall thickness of the left ventricle, as shown by gadolinium-based magnetic resonance [8]. Heart valves are involved in the disease. Typical thickening of the aortic and mitral valve flaps may be significantly present in both children (20\%) and adults: $57 \%$ of AFD patients show impaired mitral valve and $47 \%$ show alteration of the aortic valve, with milder mitral prolapse. The aim of this paper is to provide deeper insights into the imaging data in AFD patients, irrespective of their genetic background.

\section{Diagnostic work-up Electrocardiography}

At the baseline ECG, shorter PR intervals are often the first (and sometimes the only) signs of cardiac involvement due to reduction of the P-wave duration. Specific features include:

A) Left ventricular hypertrophy. In adult patients, the electrocardiographic signs of LVH are present in more than $61 \%$ of males and $18 \%$ of females and are associated with repolarization abnormalities. The QRS duration is reported to be around 160-200 msec and the amplitude/duration product, in the 12-lead ECG, is correlated with the left ventricular mass, as measured both by MRI and pathological findings (post-mortem).
B) PR interval. The involvement of conduction tissue, due to the progressive infiltration by Gb3, leads to the onset of arrhythmias, showing a characteristic electrocardiographic framework. Depending on age and progression of the disease, PR is shorter in affected males (up to $11 \%$ ) [3, 9]. With progression of the disease, conduction time may increase and the ECG may show a ventricular atrium block (VAB).

\section{Cardiac imaging}

A) Echocardiography LV hypertrophy is the morphological characteristic of the disease, often accompanied by myocardial fibrosis. A concentric hypertrophy usually occurs in adulthood of AFD patients with fibrosis of the left-lateral wall of the LV. However, eccentric hypertrophy can be detected too, as a result of the thinning of the left ventricular back-lateral basal segment, due to prevalence of the fibrous substitution. A 'binary' aspect of the endocardium - first detected and described by Pieroni et al. [2], may evoke the presence of AFD in subjects with LVH. Figure 1a, b, c and Video. Thickening of the papillary muscles, with or without hypertrophy of the left ventricular walls, is also prominent in these patients so that the ratio between papillary muscle size to LV circumference has been proposed [3] as an echocardiographic marker for diagnosis of AFD. Left ventricular ejection fraction (EF) is usually normal and when reduced, mortality increases. Cardiomyopathy in AFD patients has often been labeled in association with restrictive diastolic dysfunction but a restrictive pattern has proved to be extremely rare. In general, ventricular relaxation phase abnormalities are common, whereas a pseudo-normalization of trans-mitral doppler is observed in more advanced stages. Tissue Doppler Imaging (TDI) with strain deformation 

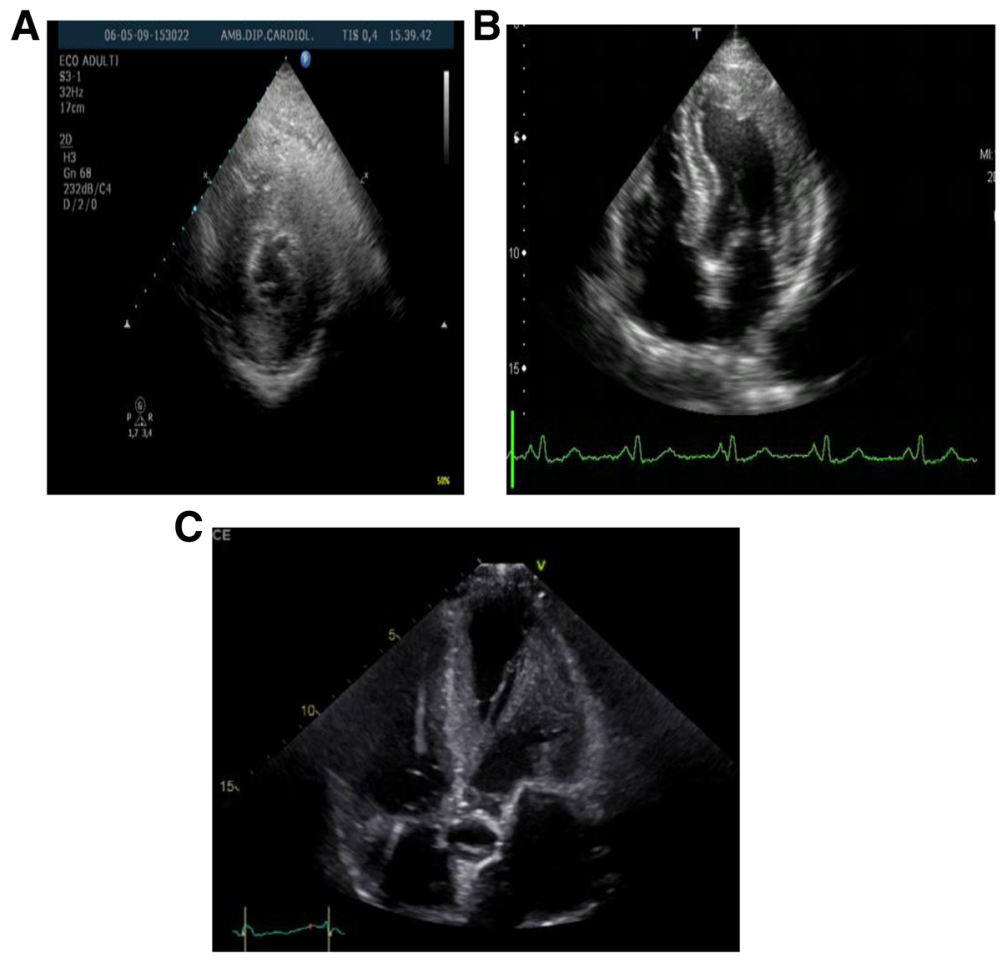

Fig. 1 a, b, c Video. Short left echocardiogram (left) and 4-chamber apical (right) highlights the presence of concentric ventricular hypertrophy

analysis, Zamorano et al. [10, 11] showed that velocities of the septum and lateral segment of the mitral anulus were inversely proportional to the left ventricular mass in a cohort of AFD patients. Fig. 2a,b In our series, strain rate imaging has been proven to differentiate sarcomeric HCM from AF cardiomyopathy or even cardiac amyloidosis . [12] Shank et al. [13] showed that in 16 patients, AFD had reduced longitudinal systolic strain, while there were no differences in circumferential systolic strain. Diastolic function assessment showed reduced longitudinal early diastolic $S R, S_{I V R}$, and $\mathrm{E} / \mathrm{SR}_{\mathrm{IVR}}$ (SR(IVR) $(P<.001)$ and E/SR(IVR) $(P=.025)$ remained significantly different between patients with FD and controls, with sensitivity of $94 \%$ and specificity of $92 \%$ for SR(IVR) of $0.235 \mathrm{~s}(-1)$ (area under the receiver operating characteristic curve, 0.953), while radial and circumferential diastolic function are not affected. Right ventricle involvement is largely parallel to the left ventricle, although it is interesting to note that the right ventricle develops fibroblast hypertrophy, as evaluated with cardiac magnetic resonance imaging [14]. Systolic excursion of tricuspid annulus (TAPSE) is the standard parameter for monitoring the function of the right ventricle and this often appears to occur normally, even in advanced stages of the disease.
Valve abnormalities are frequently observed, but alterations are usually mild, although Gb3 accumulation is present in valves.

B) Cardiovascular magnetic resonance or magnetic resonance imaging (CMR/MRI).

CMR is helpful in diagnosing HCM by identifying areas of hypertrophy, not visualized by echocardiography, providing a differential diagnosis of HCM from other causes of LVH [15]. Infiltrative cardiomyopathies, including glycogen/lysosmal storage disease, can mimic HCM as they can produce increased wall thickness. Differentiation of these 'phenocopies' is critical, as treatment strategies and prognosis differ compared with HCM. [16] . T1 mapping, a novel CMR imaging technique, has a great deal of power by providing further differentiation of HCM from these infiltrative cardiomyopathies. Fig. 3a,b. [17]. T1 mapping of the LV was performed in a basal and mid-ventricular short axis slice using the SAturation-recovery single-SHot Acquisition (SASHA) SSFP pulse sequence. [18] This tool has proved to be powerful in the diagnosis of cardiac involvement in AFD and also in providing insight into the biology of the pathology itself. T1 mapping is a more recent advanced tissue characterization technique whereby a low T1 represents sphingolipid deposition. A low T1 occurs in Anderson-Fabry 

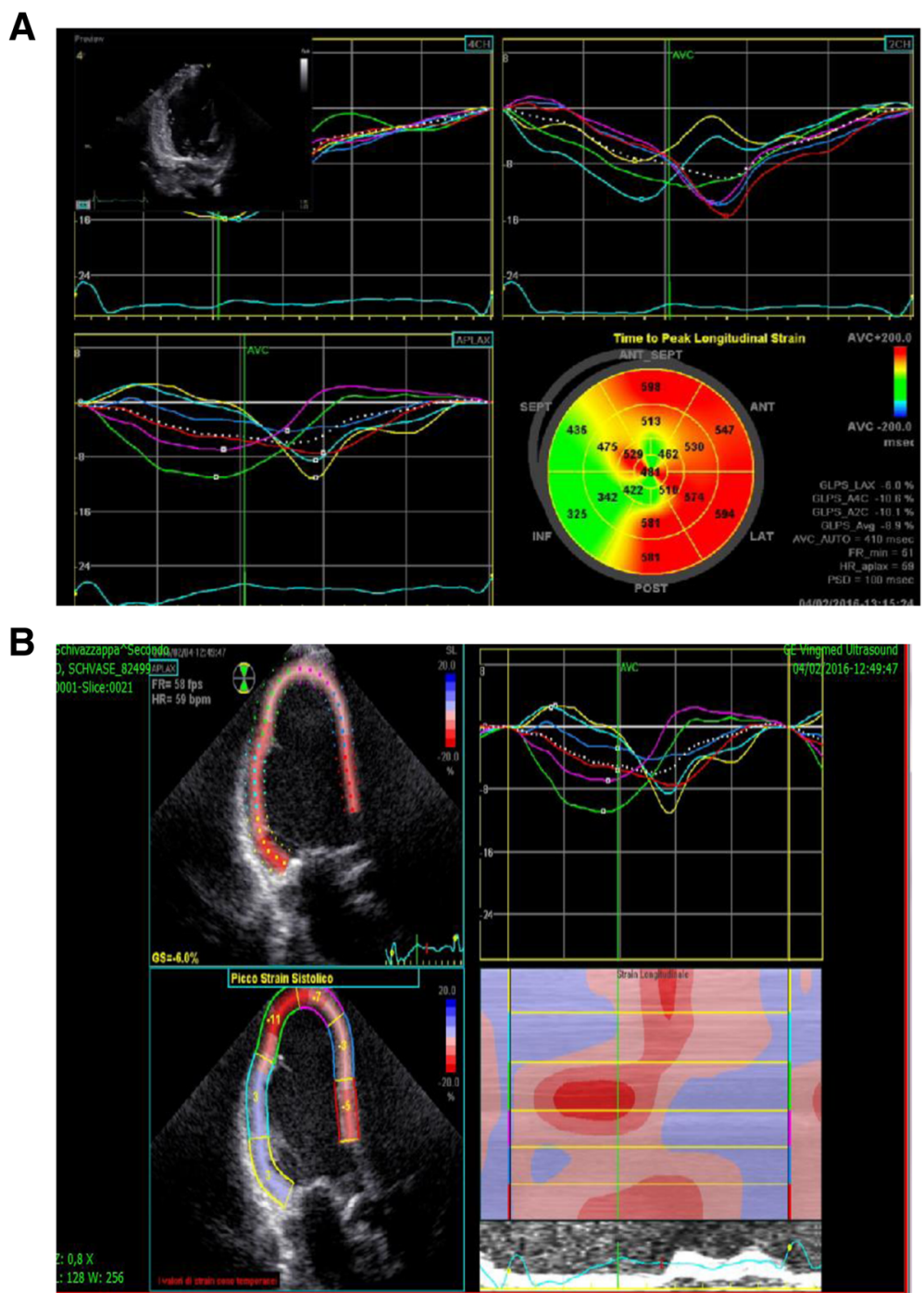

Fig. 2 a-b Left Ventricle with low longitudinal strain deformation

cardiomyopathy associated with an increase of the extracellular volume (ECV), but not in amiloid infiltrative cardiomyopathy (AL,TTN) $[19,20]$. In our series, CMR has proved to be the gold standard at measuring cardiac mass and function, but it's strength is in tissue characterization. Late gadolinium enhancement in the classic location of the basal inferolateral wall is identified in up to $50 \%$ of AFD patients. It occurs in males with $\mathrm{LVH}$, and females with or without LVH. It represents fibrosis in an advanced stage of the disease. Recent studies suggest that it also represents inflammation using T2 mapping and biomarkers [20]. CMR provides important supplemental information on tissue characterization using gadolinium, and provides differential diagnosis aspects among the various hypertrophic cardiomyopathies. In particular, a relaxation time (T2) is typical in patients with hypertrophic sarcomeric cardiomyopathy and hyper-enhancement relief at the left ventricular wall of the left ventricle. The extension of hypertrophy of cardiomyocytes and the accumulation of Gb3 within vacuums correlates with the extension of the wall thickness of the left ventricle, visible on gadolinium-based magnetic resonance [19].

\section{Conclusion}

Anderson-Fabry disease, a rare X-linked disorder, is a systemic lysosomal disorder. AFD cardiomyopathy can mimic hypertrophic sarcomeric cardiomyopathy (HCM) or amyloid cardiomyopathy. Differential diagnosis, by multidisciplinary approach and by incorporating specific cardiac signs, has important therapeutic and prognostic implications because AFD patients have benefited from 
A

B

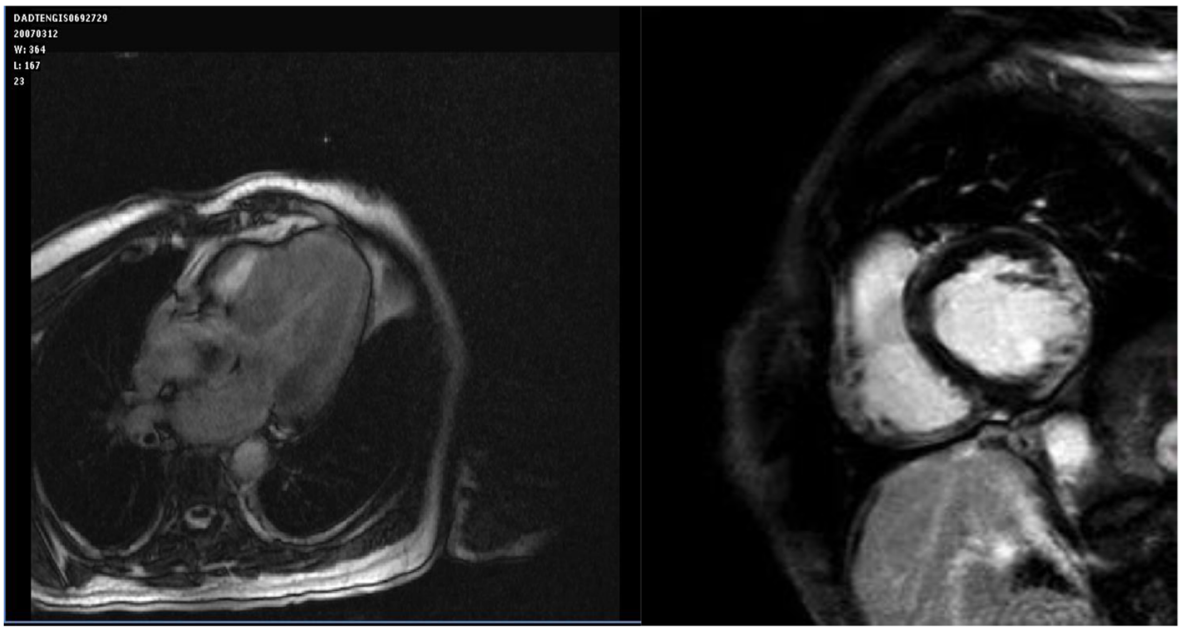

Fig. 3 a, b Cardiac Magnetic Resonance shows a typical hypertrophic cardiomyopathy with hyper-enhancement relief at the left ventricular wall and enhancement relief at the left ventricular wall

an enzyme replacement therapy (ERT) since 2001. Cardiac imaging plays an important role in detecting this peculiar disease in patients with cardiac septum/wall thickening, both in the classic AFD and in its cardiomyopathy variant (AFD cardiomyopathy) form, therefore, thanks to timely diagnosis, avoiding end-stage renal failure and heart failure.

\section{Abbreviations}

AFD: Anderson-Fabry Disease; AVB: Atrio-Ventricular Block; EF: Left ventricular Ejection Fraction; ERT: Enzyme Replacement Therapy; LVCH: Left Ventricle Cardiac Hypertrophy; LVM: Left Ventricular Mass; PM: Pace-maker;

TAPSE: Systolic excursion of tricuspid annulus; TDI: Tissue Doppler Imaging

\section{Acknowledgements}

I thank Mr. A. Bonetto (e-mail ibonetto@alice.it and Mrs. B. Serra (e-mail barbara.serra@alice.tt for contributing the English review and Mrs. R. Bandini, librarian (e-mail rina.bandini@unipr.it), Department of Physics University of Parma for helping to prepare video-clips and to supply bibliographic research.

\section{Availability of data and materials}

All data generated or analyzed during this study are available from the corresponding author or reasonable request.

\section{Authors' contributions}

WS is the major contributor in writing the manuscript and prepared the images; NM was involved in revising manuscript critically for important intellectual content; WS made substantial contributions to conception and design, interpretation of data and was involved in giving final approval of the version to be published. NM reviewed the genetic part. Both authors read and approved the final manuscript.

\section{Authors' information}

Not applicable.

\section{Ethics approval and consent to participate}

Not available.

\section{Competing interests}

The authors declare that they have no competing interests.

\section{Publisher's Note}

Springer Nature remains neutral with regard to jurisdictional claims in published maps and institutional affiliations.

\section{Author details}

${ }^{1}$ Cardiology Division, Surgery Department, University Hospital-Parma, Via Antonio Gramsci 14, 43100 Parma, IT, Italy. ${ }^{2}$ University of Molise, Health Sciences Department-Campobasso, Campobasso, IT, Italy. ${ }^{3}$ Fondazione Floresta Longo, Catania, IT, Italy.

Received: 15 October 2018 Accepted: 14 January 2019

Published online: 23 January 2019

\section{References}

1. 2014 ESC Practice Guidelines on diagnosis and management of hypertrophic cardiomyopathy Eur Heart Journal (2014) 35:2733-2779 https://doi.org/10.1093/eurheartj/ehu284

2. Pieroni M, Chimenti C, De Cobelli F, et al. Fabry's disease cardiomyopathy: echocardiographic detection of endomyocardial glycosphingolipid compartmentalization. J Am Coll Cardiol. 2006;47:1663-71.

3. M N, Liu D, Hu K, Herrmann S, Breunig F, Strotmann J, Störk S, Voelker W, Ertl G, Wanner C, Weidemann F. Prominent papillary muscles in Fabry disease: a diagnostic marker? Ultrasound Med Biol. 2011 jan;37(1):37-43. https://doi.org/10.1016/j.ultrasmedbio.2010.10.017.

4. Serra W, Fagnani S, Ardissino D, Gherli T. Late-onset cardiac variant of Fabry disease responsive to short-term treatment with Agalsidase alpha. J Clinic Experiment Cardiol. 2010;1:109. https://doi.org/10.4172/2155-9880.1000109.

5. Laney DA, et al. Fabry disease practice guidelines: recommendations of the National Society of genetic counselors. J Genet Couns. 2013;22(5):555-64.

6. Eng $\mathrm{CM}$, et al. Fabry disease: guidelines for the evaluation and management of multi-organ system involvement. Genet Med. 2006;8(9):539-48.

7. Pieruzzi $F$, et al. Heart involvement in Anderson-Fabry disease: Italian recommendations for diagnostic, follow up and therapeutic management. G Ital Cardiol (Rome). 2015;16(11):630-8.

8. Gange CA, Link MS, Maron MS. Utility of Cardiovascular Magnetic Resonance in the Diagnosis of Anderson-Fabry Disease. Circulation. 2009; 120:e96-7.

9. Mehta A, Ricci R, Widmer U, et al. Fabry disease defined: baseline clinical manifestations of 366 patients in the Fabry outcome survey. Eur J Clin Investig. 2004;34:236-42.

10. Zamorano J, Serra V, Perez de Isla L, et al. Usefulness of tissue Doppler on early detection of cardiac disease in Fabry patients and potential role of enzyme replacement therapy (ERT) for avoiding progression of disease. Eur J Echocardiogr. 2011;12:671-7. 
11. F. Weidemann a, A. Linhart b, L. Monserrat c, J. Strotmann . Cardiac challenges in patients with Fabry disease. IJC (2009), doi:10.1016/j.jicard. 2009.08.00.

12. Sun JP, Stewart WJ, Yang XS, Donnell RO, Leon AR, Felner JM, Thomas JD, Merlino JD. Differentiation of hypertrophic cardiomyopathy and cardiac amyloidosis from other causes of ventricular wall thickening by twodimensional strain imaging echocardiography. Am J Cardiol. 2009;103:4115. https://doi.org/10.1016/j.amjcard.20.

13. Shanks M MD Thompsonlan RB . Paterson ID, Oudit GY. MD, PhD Systolic and diastolic function assessment in Fabry disease patients using speckletracking imaging and comparison with conventional echocardiographic measurements. J Am Soc Echocardiogr 2013;26(12):1407-1414. doi: https:// doi.org/10.1016/j.echo.2013.09.005.

14. Kampmann C, Baehner FA, Whybra C, et al. The right ventricle in Fabry disease. Acta Paediatr Suppl. 2005:94:15-8 discussion 9-0.

15. Rowin EJ, Maron MS. The role of cardiac MRI in the diagnosis and risk stratification of hypertrophic cardiomyopathy. Arrhythm Electrophysiol Rev. 2016;5(3):197-202. https://doi.org/10.15420/aer.2016:13:3.

16. Maceira AM, Joshi J, Prasad SK, et al. Cardiovascular magnetic resonance in cardiac amyloidosis. Circulation. 2005;111:186-93. https://doi.org/10.1161/01. CIR.0000152819.97857.9D.

17. Moon JC, Sachdev B, Elkington AG, et al. Gadolinium enhanced cardiovascular magnetic resonance in Anderson-Fabry disease. Evidence for a disease specific abnormality of the myocardial interstitium. Eur Heart $\rfloor$. 2003;24:2151-5.

18. Tham EB, Chow K, Spavor M, Pagano JJ, Haykowsky M, Thompson RB. Degree ofdiffuse fibrosis measured by mri correlates with Iv remodelling in childhood cancer survivors after anthracycline chemotherapy. J Cardiovasc Mag Reson. 2011;13(Suppl1):276.

19. T1 mapping in Cardiac MRI D.Radencovich,S Weingarthner,G Captur Heart Fail Review 2017, 22:415-430.

20. Sabrina Nordin, Rebecca Kozor, Heerajnarain Bulluck, Silvia Castelletti, Stefania Rosmini, Amna et al .Cardiac Fabry disease with late gadolinium enhancement is a chronic inflammatory cardiomyopathy. JACC Volume 68, Issue 15, October 2016 DOl: https://doi.org/10.1016/j.jacc.2016.07.741.

Ready to submit your research? Choose BMC and benefit from:

- fast, convenient online submission

- thorough peer review by experienced researchers in your field

- rapid publication on acceptance

- support for research data, including large and complex data types

- gold Open Access which fosters wider collaboration and increased citations

- maximum visibility for your research: over $100 \mathrm{M}$ website views per year

At $\mathrm{BMC}$, research is always in progress.

Learn more biomedcentral.com/submissions 